\title{
Microwave Irradiation-Assisted Chitosan Hydrolysis Using Cellulase Enzyme
}

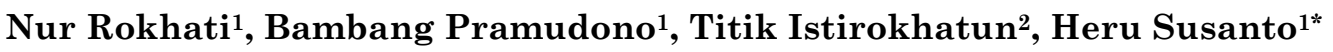 \\ ${ }^{1}$ Department of Chemical Engineering, Diponegoro University, Jl. Prof. Soedarto, Kampus Undip \\ Tembalang, Semarang 50239, Indonesia \\ ${ }^{2}$ Department of Environmental Engineering, Diponegoro University, Jl. Prof. Soedarto, Kampus Undip \\ Tembalang, Semarang 50239, Indonesia
}

Received: $19^{\text {th }}$ March 2018; Revised: $19^{\text {th }}$ June 2018; Accepted: $25^{\text {th }}$ June 2018; Available online: $14^{\text {th }}$ November 2018; Published regularly: December 2018

\begin{abstract}
The influence of microwave irradiation on the chitosan hydrolysis catalyzed by cellulase enzyme was studied. The hydrolyzed chitosan was characterized by measuring its viscosity and reducing sugar. Further, it was also characterized by Fourier-Transform Infrared Spectroscopy (FT-IR), X-ray Diffraction (XRD), and Scanning Electron Microscope (SEM). The classical Michaelis-Menten kinetic parameters were measured by analyzing the amount of reducing sugars. The results were compared with the hydrolysis by using conventional shaker incubator. The hydrolysis reaction time needed to obtain similar reducing sugar yield was significantly lower for microwave irradiation than shaker incubator. On the other hand, the reduction rate of the relative viscosity was significantly higher for the hydrolysis of chitosan using shaker incubator. A significant difference in chemical structure was observed between hydrolysis using microwave irradiation and shaker incubator. Overall, the result showed that the hydrolysis behavior of chitosan using microwave irradiation is significantly different with using shaker incubator. Copyright (C) 2018 BCREC Group. All rights reserved
\end{abstract}

Keywords: Microwave Irradiation; Hydrolysis; Chitosan; Cellulase

How to Cite: Rokhati, N., Pramudono, B., Istirokhatun, T., Susanto, H. (2018). Microwave IrradiationAssisted Chitosan Hydrolysis Using Cellulase Enzyme. Bulletin of Chemical Reaction Engineering \& Catalysis, 13 (3): 466-474 (doi:10.9767/bcrec.13.3.2378.466-474)

Permalink/DOI: https://doi.org/10.9767/bcrec.13.3.2378.466-474

\section{Introduction}

Chitosan is a natural polysaccharide composed of D-glucosamine and N-acetyl-Dglucosamine with $\beta$ (1-4) linkage. Unlike most polysaccharides, the presence of free electron pair of the amine group, which acts as a cation allows chitosan for binding to compounds that are anionic by an ionic or a hydrogen bond [1,2]. Chitosan has the properties of biocompatibility,

\footnotetext{
* Corresponding Author.

E-mail: heru.susanto@che.undip.ac.id (H. Susanto)

Telp: +62-24-7460058, Fax: +62-24-76480675
}

degradable, bioactivity, and non-toxicity. Low molecular weight chitosan (LMWC) has been widely used in many fields, such as food [3], agriculture [4], biomaterials [5], and pharmaceutical $[6,7]$ industries. Chitosan is synthesized by deacetylation of the chitin. In general, chitosan has high molecular weight, causing poor solubility at neutral $\mathrm{pH}$ and high viscosity aqueous solutions. This property inhibits wider application of chitosan.

Low molecular weight chitosan (LMWC) has been prepared by a variety of techniques, including acid hydrolysis, oxidative degradation, 
and enzymatic methods. The high acid concentration is used in acid hydrolysis of chitosan, which requires special equipment with high corrosion resistance and brings major waste disposal problems [8]. The oxidative degradation may cause some changes in its chemical structure [9]. The enzyme hydrolysis receives more attention for its mild reaction conditions, more specific, and allows a control of the extent of reaction [10]. However, enzymatic hydrolysis has a very low reaction rate. As reported by Roncal et al. [10], Cabrera et al. [11], and Lee et $a l$. [12], that the chitosan enzymatic hydrolysis incubator required more reaction time.

In recent years, the microwave irradiation as a non-conventional energy source has received increasing attention in organic chemistry because of its ability to improve the selectivity, accelerate of reaction, and increase product yield compared to conventional heating $[13,14]$. Li et al. [15] studied oxidative degradation of chitosan by $\mathrm{H}_{2} \mathrm{O}_{2}$ under microwave irradiation. They found that the rate of degradation increased more than five times as compared to conventional heating without microwave irradiation. Saxena et al. [16] have performed hydrolysis of triolein under microwave irradiation. The enzymatic reaction rate of triolein was increased. Thus, the microwave is proven to increase the reaction rate and enzyme resistant to a microwave irradiation. To the best of our knowledge study of microwaveassisted chitosan enzymatic hydrolysis has not been conducted in previous publications.

In this study, chitosan hydrolysis using cellulase enzyme under a microwave irradiation was investigated to increase the reaction rate of the hydrolysis. Therefore, the research aims to study the effect of microwave irradiation on the behavior of chitosan hydrolysis using cellulase enzyme. Furthermore, comparison of microwave irradiation-assisted chitosan hydrolysis and shaker incubator-assisted chitosan hydrolysis was performed.

\section{Materials and Methods}

\subsection{Materials}

Raw chitosan was purchased from PT. Biotech Surindo, Cirebon, Indonesia. The characterization of this raw chitosan showed that the viscosity was $375.5 \mathrm{cps}(1 \%$ chitosan solution in $1 \%$ acetic acid solution) and degree of deacetylation (DD) was 85.78 . The protein, water, and ash contents were $0.5 \%, 5.1 \%$, and 1.2 $\%$, respectively. The cellulase enzyme was purchased from Sigma-Aldrich Germany (Cat. 22178 ) and its average activity was $\sim 0.8 \mathrm{U} / \mathrm{mg}$.
Glacial acetic acid $\left(\mathrm{CH}_{3} \mathrm{COOH}\right)$ and sodium carbonate $\left(\mathrm{Na}_{2} \mathrm{CO}_{3}\right)$ were purchased from Merck KGaA Germany. Potassium ferricyanide $\left(\mathrm{K}_{3}\left(\mathrm{Fe}(\mathrm{CN})_{6}\right)\right)$ was purchased from SigmaAldrich, Germany.

\subsection{Hydrolysis of Chitosan}

Chitosan was dissolved in acetic acid/ sodium acetate buffer $(0.1 \mathrm{M})$ at concentration of $0.5 \%$ and $1 \%(\mathrm{w} / \mathrm{v})$. All degradation studies were performed in a $100 \mathrm{~mL}$ Erlenmeyer flask. Chitosan solution $(50 \mathrm{~mL})$ was treated with cellulase in the ratio of 100:1 (w/w). The hydrolysis using conventional shaker incubator was carried out at the optimum process of $50{ }^{\circ} \mathrm{C}$ [17] and $100 \mathrm{rpm}$. Samples were withdrawn at every 15 min during the first hour. Then, the next samples were withdrawn at every $30 \mathrm{~min}$ up to a $3^{\text {rd }}$ hour and every 1 hour up to $8^{\text {th }}$ hour. The last sample was withdrawn after 24 hours.

The procedure for chitosan hydrolysis using microwave irradiation was almost similar with shaker incubation. The hydrolysis using microwave irradiation was carried out with the power of 40 and 120 watts. Samples were withdrawn from the first minute up to $10 \mathrm{~min}$. The reaction was stopped by heat inactivation of the enzyme in a boiling water bath for $10 \mathrm{~min}$. Then the samples were subjected to viscosity and reducing sugars analysis. A part of samples was adjusted to $\mathrm{pH} 8.0$ with $10 \% \mathrm{NaOH}$ solution, resulting in a precipitate, and oven dried prior to FTIR, XRD, and SEM analysis.

\subsection{Analysis}

Relative viscosity was measured as the decrease in viscosity of the chitosan solution using Ostwald viscometer at $28 \pm 1{ }^{\circ} \mathrm{C}$ and determined using Equation (1) [10].

$$
\eta_{\text {rel }}=t / t_{0}
$$

where, $\eta_{\text {rel }}$ is relative viscosity, $t$ is the flow time of chitosan sample solution, and to is the flow time of initial chitosan solution in the absence of hydrolysis.

Analysis of total reducing sugars was conducted according to Imoto method [9]. The Imoto reagent was prepared as follows: $0.50 \mathrm{~g}$ of potassium ferricyanide was mixed with $1 \mathrm{~L}$ of $0.5 \mathrm{M}$ sodium carbonate solution and then was stored in a glass bottle protected from light. A mixture containing $4 \mathrm{~mL}$ of Imoto reagent and $3 \mathrm{~mL}$ of sample was heated for $15 \mathrm{~min}$ at 100 ${ }^{\circ} \mathrm{C}$ followed by cooling to room temperature. 
The absorbance of the mixture solution was measured at $420 \mathrm{~nm}$. Total reducing sugars were calculated based on a standard curve obtained with $\mathrm{D}$-glucosamine $\mathrm{HCl}$.

FTIR spectra were recorded with $\mathrm{KBr}$ powder using the instrument IR Prestige-21 Shimadzu, Japan. Thirty-two scans were performed at a resolution of $4 \mathrm{~cm}^{-1}$ and a temperature of $21 \pm 1{ }^{\circ} \mathrm{C}$ over the wavelength range of $500-4,000 \mathrm{~cm}^{-1}$. XRD patterns were obtained by a Shimadzu Lab XRD - 7,000 diffractometer and use a CuKa target at $30 \mathrm{kV}$ and $30 \mathrm{~mA}$ at 20 ${ }^{\circ} \mathrm{C}$. The scattering range $(2 \theta)$ was recorded from 5 to 400 . The relative crystallinity (RC) of chitosan was calculated using Equation (2) [18].

$$
R C(\%)=(A c /(A c+A a)) * 100
$$

where, $A c$ is the crystalline area and $A a$ is the amorphous area. The morphology of chitosan was examined using a scanning electron microscope (JEOL JSM-6510LA SEM, Japan) at an acceleration voltage of $10 \mathrm{kV}$. The sample was coated with platinum and a magnification of $10,000 \mathrm{X}$.

\section{Results and Discussion}

3.1 The Effect of Hydrolysis Reaction on Viscosity and Reducing Sugar

The change in the viscosity of the linear polysaccharide solution by enzymatic degradation reflects the change in the degree of polymerization [11]. Thus, the course of degradation of chitosan samples was conveniently studied by viscosity measurement, and characterized by the decrease of the relative viscosity at a given time of degradation $[11,19]$. The rela- tive viscosity as a function of incubation time on chitosan sample concentration of $0.5 \%$ and $1 \%(\mathrm{w} / \mathrm{v})$ under the shaker incubator are shown in Figure 1A.

The relative viscosity of the reaction mixture dropped dramatically in the early reaction stage. The hydrolysis of the initially long chitosan chains caused greater viscosity decreases than the subsequent degradation of their shorter hydrolysis products. Fast degradation during the initial hydrolysis stage is attributed to endo-enzyme activity [20]. After $4 \mathrm{~h}$, degradation slowed down. This phenomenon is probably due to the inhibition of the enzyme activity by the end products [19].

Each enzymatic cleavage of relatively rigid linear polysaccharides generates the new reducing sugar. The formation of reducing sugar indicates the activity of an enzyme that cleaves glycosidic bond in chitosan polymer. The effect of hydrolysis reaction time on reducing sugar formation are shown in Figure 1B. It shows that within the first hour, a relatively fast formation of reducing sugar was observed. After that, decreasing their formation rates in the following $4 \mathrm{~h}$ was observed. The formation of reducing sugar of chitosan samples seemed to be constant after $8 \mathrm{~h}$ of hydrolysis.

Chitosan degradation in $0.1 \mathrm{M}$ acetic buffer solution with cellulase enzyme under microwave irradiation was investigated. Figure 2A shows that the rate of the relative viscosity decrease is significantly lower compared with shaker incubator. Then, the formation rate of reducing sugar is significantly higher for microwave irradiation (Figure 2B). Under the condition of microwave irradiation, the yield
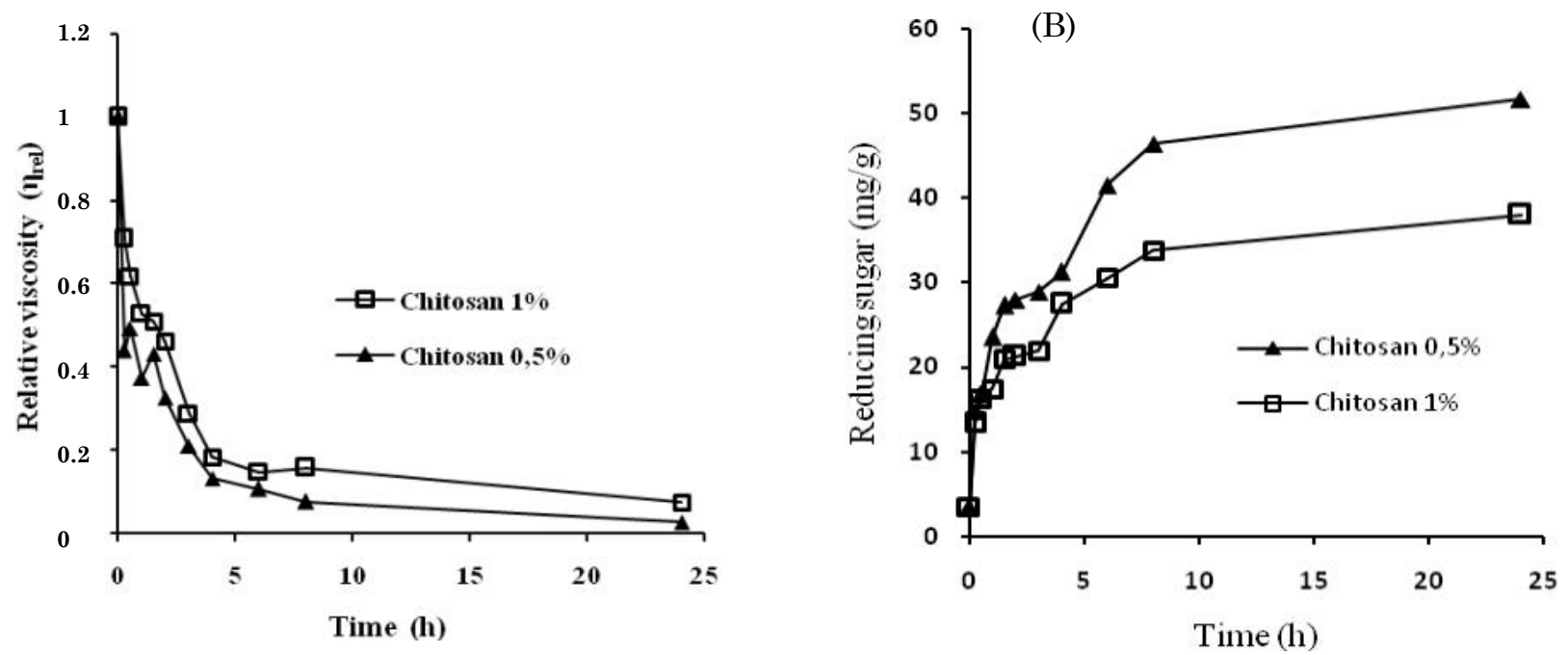

Figure 1. The effect of reaction time at chitosan hydrolysis using shaker incubator (temperature 50 ${ }^{\circ} \mathrm{C}, 100 \mathrm{rpm}$ ) on relative viscosity (A) and reducing sugar (B) 
reducing sugar is approximately $25 \mathrm{mg} / \mathrm{g}$ after 10 min hydrolysis. By contrast, to obtain similar yield (25 mg/g), it is required 3 hour for hydrolysis using shaker incubator (Figure 1B). It can be explained that microwave irradiation induces polar molecules. The oscillating electric field from microwaves creates friction among molecules resulting in fast heating [21]. In addition, although the microwave irradiation only contains one joule per mole of photons, it can reduce activation of the Gibbs energy of reaction through microwave energy storage in the vibrational energy via effect enthalpy and entropy of molecule [22]. Consequently, the activation reaction in a polar bond such as glycoside bond increased and the cleavage of glyco-

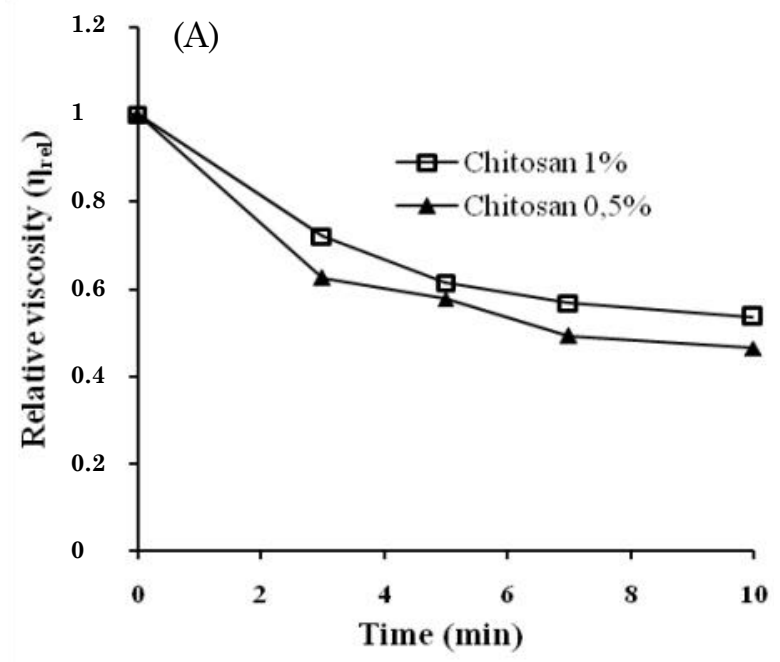

sidic bond occurs on the reducing end of chitosan [15]. Thus, this phenomenon causes the reduction rate of the relative viscosity decreased and the formation rate of reducing sugars increased. This is in accordance with the characteristics of the exo-type reaction that has been described by Lee et al. [12] and Li et al. [19].

The degradation of chitosan under microwave irradiation with different power was studied. The effect of hydrolysis reaction time at different power on relative viscosity and reducing sugar are shown in Figure 3. The relative viscosity decreased (Figure $3 \mathrm{~A}$ ) and the reducing sugar formation increased (Figure 3B) with increasing power. This phenomenon can

Figure 2. The effect of reaction time at chitosan hydrolysis using microwave irradiation (power 40 watt) on relative viscosity (A) and reducing sugar (B)
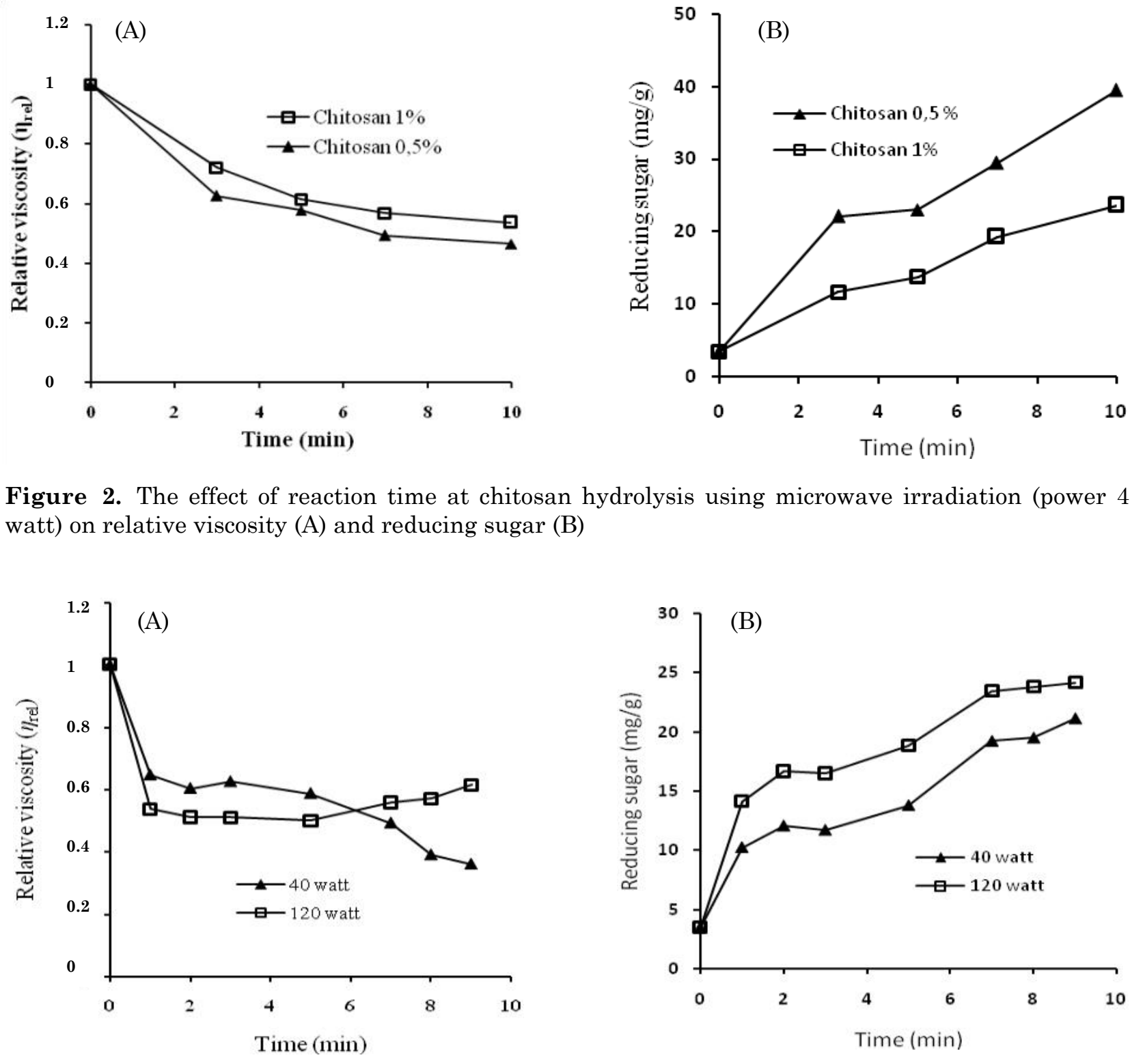

Figure 3. The effect of hydrolysis reaction time at different microwave power on relative viscosity (A) and reducing sugar (B) 
be explained that increasing power can increase the resulting photons thus the cleavage rate of a glycosidic bond increased. This statement is well explained by Mello [21]. However, the relative viscosity increased after 5 minutes at a power of 120 watts (Figure 3A). This increase can be explained that the heating rate of material increases with increasing microwave power. Because the solvent is easily evaporated, therefore the viscosity increased.

The reducing sugar yield of degraded chitosan products increased with increasing power. The degraded products with similar reducing sugar yield required shorter reaction time at high power level than at low power level (Figure 3B). Under a microwave irradiation field, the intensified molecular motions and collisions enable a high probability of contact between substrate and enzyme, resulting in an enhanced reaction rate with increasing power.
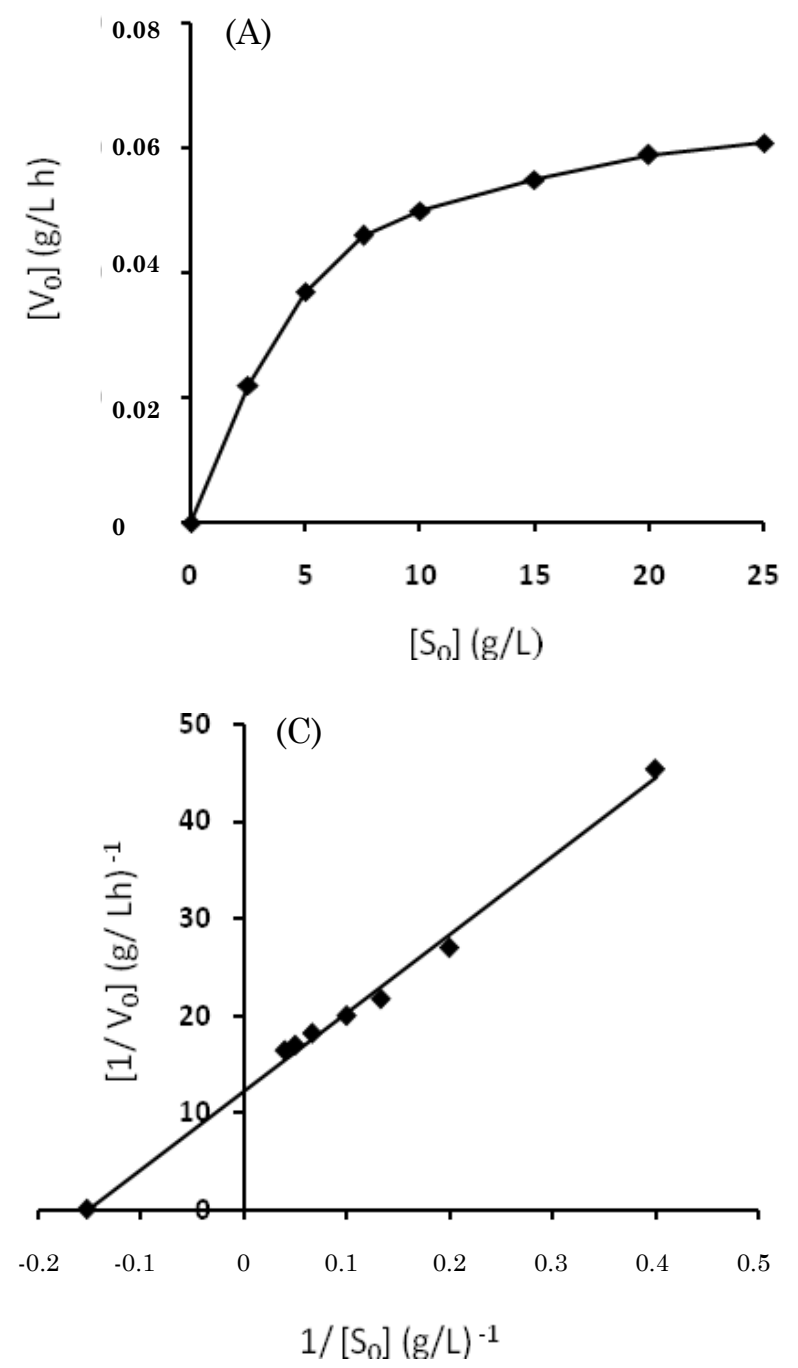

This statement similar to the previously publication by Li et al. [15] and $\mathrm{Su}$ et al. [23].

\subsection{Kinetics of the Chitosan Hydrolysis}

Michaelis-Menten kinetic parameters (Equation (3)) were measured by analyzing the amount of reducing sugars. Kinetics of enzymatic hydrolysis of chitosan were investigated using varying initial concentration of the chitosan with using shaker incubator (at $50{ }^{\circ} \mathrm{C}$ and $100 \mathrm{rpm}$ ), and microwave irradiation (at power of 40 watt).

$$
\frac{1}{V}=\frac{K_{m}}{V_{\max }} \frac{1}{[S]}+\frac{1}{V_{\max }}
$$

where, $V$ is the reaction rate, $V_{\max }$ is the maximum reaction rate, $K_{m}$ is the MichaelisMenten constant, and [S] is substrate concentration.
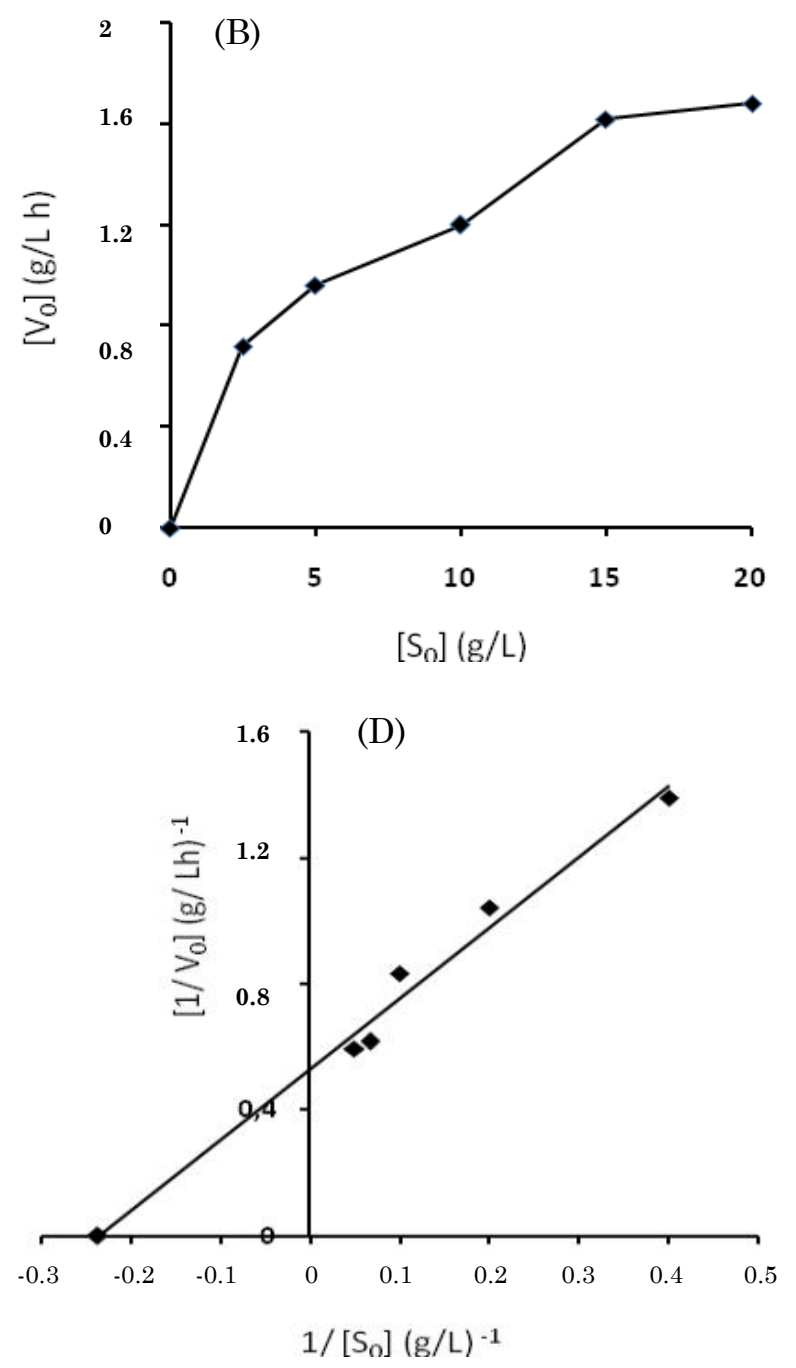

Figure 4. The effect of substrat concentration on initial velocity of reducing sugar formation at chitosan hydrolysis: (A) using shaker incubator, (B) using microwave irradiation. Lineweaver-Burk plots: (C) using shaker incubator, (D) using microwave irradiation 
Figure $4 \mathrm{~A}$ and $4 \mathrm{~B}$ shows the effect of varying substrate concentration on initial degradation rates. Lineweaver burk plot (Figure 4C and 4D) showed the $V_{\max }$ for irradiation microwave $(1.98 \mathrm{~g} / \mathrm{L} \mathrm{h})$ was higher than for shaker incubator $(0.0814 \mathrm{~g} / \mathrm{L} \mathrm{h})$, while $K_{m}$ for the irradiation microwave $(4.228 \mathrm{~g} / \mathrm{L})$ was lower than for shaker incubator $(6.516 \mathrm{~g} / \mathrm{L}) . K_{m}$ is the measure of the strength of enzyme-substrat complex. A higher $K_{m}$ indicates weak binding, whereas the lower value indicates strong binding [19].

\subsection{The Properties of Degraded Chitosan}

The chemical structure of raw and hydrolyzed chitosan was analyzed by FTIR spectroscopy. The spectrum of raw chitosan (Figure 5A) shows the $-\mathrm{CH}$ vibration band at wavelength of $2,900 \mathrm{~cm}^{-1}, \mathrm{CH}_{2}$ bond at wavelength of 1,423 $\mathrm{cm}^{-1}$, and $-\mathrm{CH}_{3}$ bond at wavelength of 1,379 $\mathrm{cm}^{-1}$. Furthermore, the absorption band at wavelength of $1,153 \mathrm{~cm}^{-1}$ indicates $-\mathrm{C}-\mathrm{O}-\mathrm{C}-$ glycosidic linkage between chitosan monomer. These results are in agreement with previous publications by Kumar et al. [24] and Prasertsung et al. [25].

In both hydrolyzed chitosan using shaker incubator (Figure 5B) and using microwave irradiation (Figure 5C) are not observed additional peak, but the peak intensity at wavelength of $2,900 \mathrm{~cm}^{-1}$ and $1,153 \mathrm{~cm}^{-1}$ decreases. The peak intensity at a wavelength of $1,153 \mathrm{~cm}^{-1}$ for hydrolyzed using microwave irradiation is lower than using shaker incubator. This indicated that more the cleavage of the glycosidic linkage

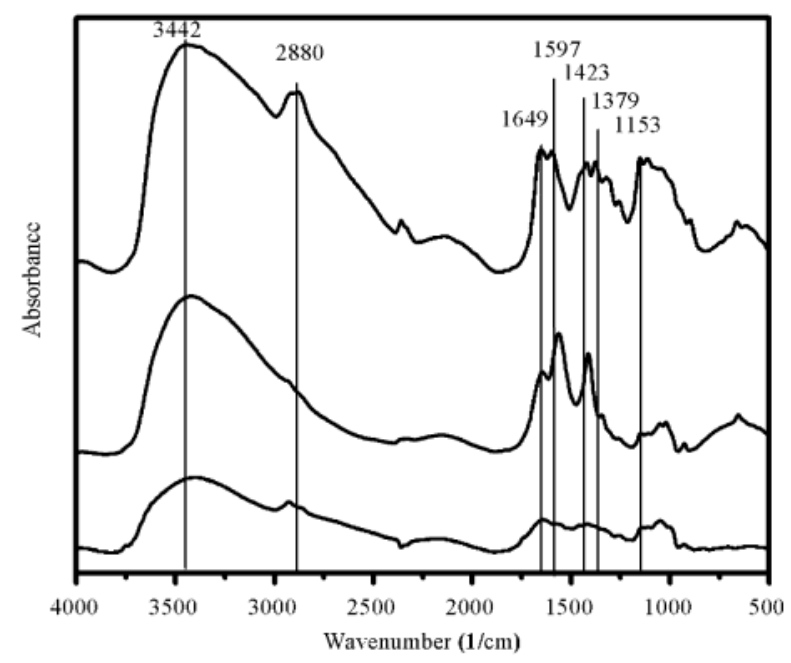

Figure 5. FTIR spectra of chitosan: (A) Raw chitosan. (B) Hydrolyzed chitosan using shaker incubator. (C) Hydrolyzed chitosan using microwave irradiation. for hydrolyzed using microwave. In hydrolyzed chitosan using microwave irradiation, the bands at wavelength of $1,410 \mathrm{~cm}^{-1}$ (symmetrical deformation of $\mathrm{CH}_{3}$ and $\mathrm{CH}_{2}$ ) and $1,370 \mathrm{~cm}^{-1}$ (bending and stretching of $\mathrm{CH}_{3}$ and $\mathrm{CH}_{2}$ ) are weak. This weakening in the absorption peak for hydrolyzed using microwave irradiation indicates a hydrogen bonding decrease in the inter-molecular and intra-molecular. This statement is similar with the previous results described by Kumar et al. [24].

The X-ray diffraction pattern of the raw chitosan, the hydrolyzed chitosan using shaker incubator, and the hydrolyzed chitosan using microwave irradiation are shown in Figure 6. The raw chitosan displayed the characteristic peaks at $2 \theta=20.40,29.30$, and 340. Compare with raw chitosan, the peak intensity of hydrolyzed chitosan at $2 \theta=20.4$ increases while at $2 \theta=$ 29.3 decreases. Hydrolysis of chitosan led to the relative crystallinity decrease. The relative crystallinity of hydrolyzed chitosan using microwave irradiation is lower than using shaker incubator. According to Li et al. [26], there is a correlation between depolymerization and crystallinity. FTIR characterization showed that there was a hydrogen bonding decrease in the inter-molecular and intra-molecular for hydrolyzed using the microwave. This is evidenced by the larger amorphous area of chitosan crystals for the hydrolyzed using microwave irradiation. Thus the crystallinity of hydrolyzed chitosan using microwave decreased causing easier to be degraded and dissolved.

Scanning electron microscope was used to visualize the morphology of raw chitosan and

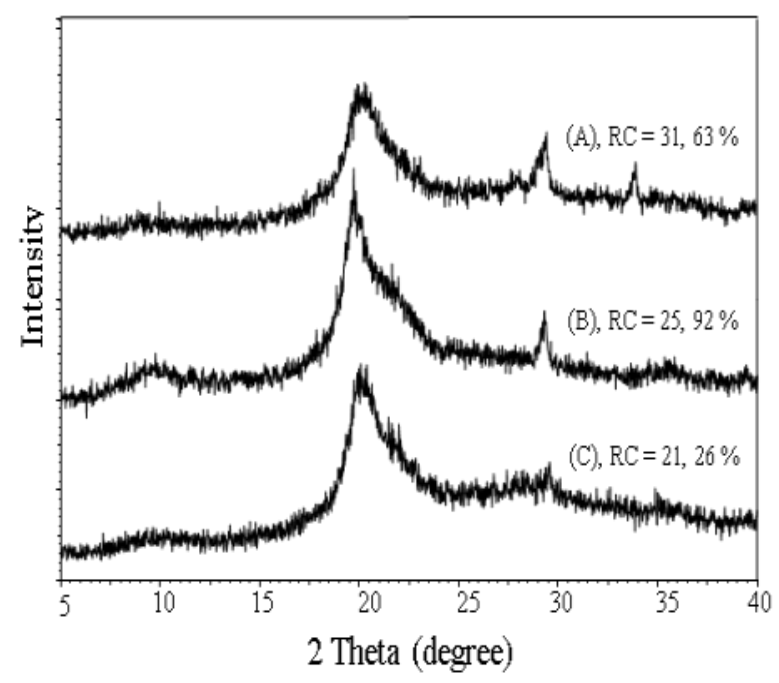

Figure 6. X-ray diffraction patterns of chitosan: (A) Raw chitosan. (B) Hydrolyzed chitosan using shaker incubator. (C) Hydrolyzed chitosan using microwave irradiation. 
hydrolyzed chitosan. The SEM results of the raw chitosan (Figure 7A) shows a non-porous, smooth, microfibrils, and crystallites, which was similar with observation by Singh et al. [27] and Lou et al. [28]. After treatment, some
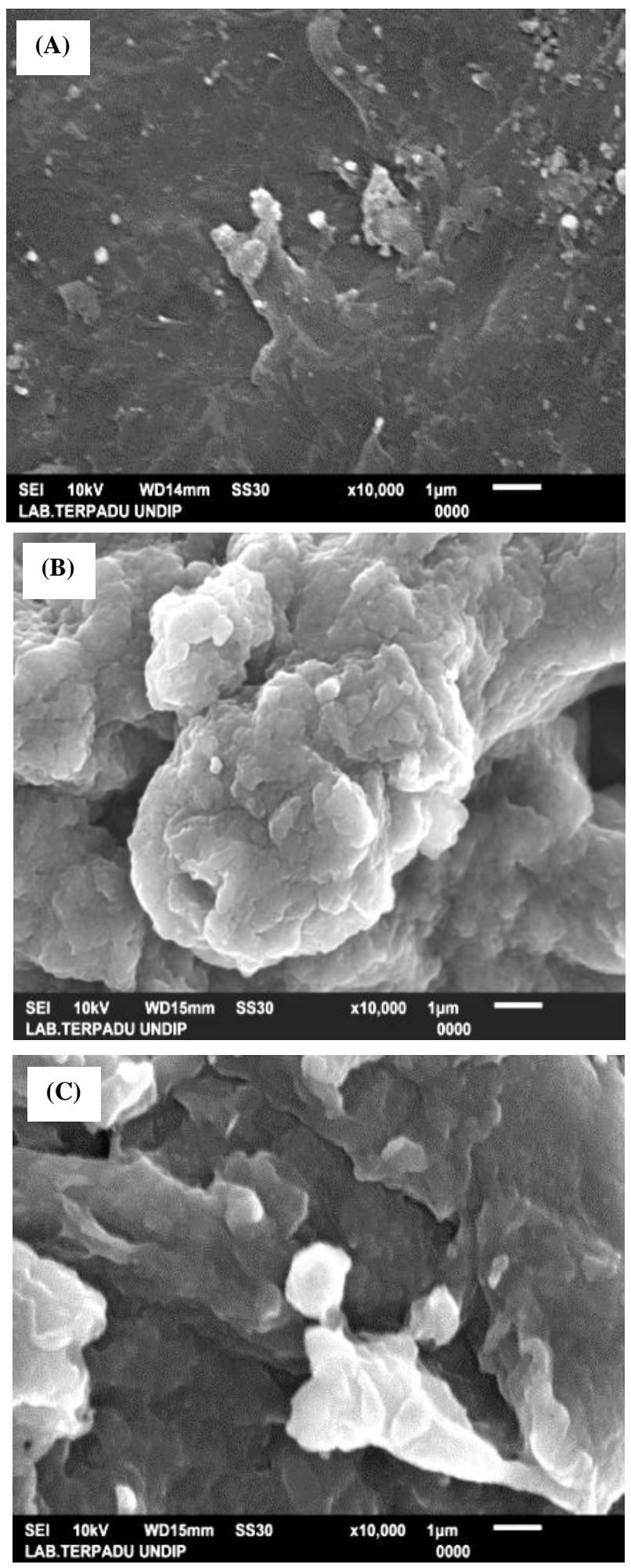

Figure 7. SEM of chitosan: (A) Raw chitosan. (B) Hydrolyze chitosan using shaker incubator. (C) Hydrolyze chitosan using microwave irradiation coarseness or pits has been generated on the surface of chitosan. The morphological differences between hydrolyzed chitosan using shaker incubator (Figure 7B) and using microwave irradiation (Figure 7C) can be observed. In the SEM results of the hydrolyzed chitosan using shaker incubator, some erosion of amorphous material from the surface of the fibers can be detected. It is relevant to the SEM result of degradation of chitosan by pulsed electric fields treatment [28]. While the SEM results of the hydrolyzed chitosan using microwave irradiation shows clear edges of the wall structure of the chitosan molecule which was broken. This indicated that the cleavage of glycosidic bond for the hydrolyzed of chitosan using microwave irradiation should occur at the end of the chitosan chain.

\section{Conclusions}

In the current study, the enzymatic hydrolysis of chitosan by using microwave irradiation was investigated and then compared with using conventional shaker incubator. The results indicated that microwave irradiation accelerates the cleavage rate of chitosan chain. The required reaction time to results in the similar reducing sugar for microwave irradiation is shorter than shaker incubator. The reducing sugar yield increases with increasing microwave power. The viscosity measurements showed that the reduction rate of the specific viscosity for the chitosan hydrolysis using shaker incubator is higher than microwave irradiation. The Michaelis-Menten kinetics showed $V_{\max }$ of $1.98 \mathrm{mg} / \mathrm{mL} . \mathrm{h}$ and $K_{m}$ of 4.228 $\mathrm{mg} / \mathrm{mL}$ for hydrolysis using the microwave irradiation, whereas $V_{\max }$ of $0.0814 \mathrm{mg} / \mathrm{mL} . \mathrm{h}$ and $K_{m}$ of $6.516 \mathrm{mg} / \mathrm{mL}$ for hydrolysis using the shaker incubator. Considering the hydrolysis location, the chemical structure of hydrolyzed chitosan using microwave irradiation should be better than hydrolyzed chitosan using shaker incubator (the end of chitosan chain vs random cutting chain chitosan).

\section{Acknowledgments}

This research was financially supported by the Ministry of Research, Technology, and Higher Education, Republic of Indonesia. The authors thank Dwi Titik Apriyanti and Haqqiyuddin Robbani for supporting the research. 


\section{References}

[1] Shahidi, F., Abuzaytoun, R. (2005). Chitin, Chitosan, and Co-Products: Chemistry, Production, Applications, and Health Effects. Advances in Food and Nutrition Research, 49: 93-135.

[2] Pillai, C.K.S., Paul, W., Sharma, C.P. (2009). Chitin and Chitosan Polymers: Chemistry, Solubility and Fiber Formation. Progress in Polymer Science, 34(7): 641-678.

[3] Prashanth, K., Tharanathan, R.N. (2007). Chitin/chitosan: Modifications and their Unlimited Application Potential-An Overview. Trends in Food Science \& Technology, 18(3): 117-131.

[4] Rinaudo, M. (2006). Chitin and Chitosan: Properties and Applications. Progress in Polymer Science, 31(7): 603-632.

[5] Huang, K.S., Wu, W.J., Chen, J.B., Lian, H.S. (2008). Application of Low-Molecular-Weight Chitosan in Durable Press Finishing. Carbohydrate Polymers, 73(2): 254-260.

[6] Dash, M., Chiellini, F., Ottenbrite, R.M., Chiellini E. (2011). Chitosan-A Versatile Semi-synthetic Polymer in Biomedical Applications. Progress in Polymer Science, 36(8): 981-1014.

[7] Anitha, A., Sowmya, S., Kumar, P.S., Deepthi, S., Chennazhi, K.P., Ehrlich, H., Tsurkan, M., Jayakumar, R. (2014). Chitin and Chitosan in Selected Biomedical Applications. Progress in Polymer Science, 39(9): 1644-1667.

[8] Tsao, C.T., Chang, C.H., Lin, Y.Y., Wu, M.F., Han, J.L., Hsieh, K.H. (2011). Kinetic Study of Acid Depolymerization of Chitosan and Effects of Low Molecular Weight Chitosan on Erythrocyte Rouleaux Formation. Carbohydrate Research, 346(1): 94-102.

[9] Zhang, Z., Li, C., Wang, Q., Zhao, Z.K. (2009). Efficient Hydrolysis of Chitosan in Ionic Liquids. Carbohydrate Polymers, 78(4): 685-689.

[10] Roncal, T., Oviedo, A., de Armentia, I.L., Fernández, L., Villarán, M.C. (2007). High Yield Production of Monomer-Free Chitosan Oligosaccharides by Pepsin Catalyzed Hydrolysis of a High Deacetylation Degree Chitosan. Carbohydrate Research, 342(18): 2750-2756.

[11] Cabrera, J.C., Van Cutsem, P. (2005). Preparation of Chitooligosaccharides with Degree of Polymerization Higher than 6 by Acid or Enzymatic Degradation of Chitosan. Biochemical Engineering Journal, 25(2): 165-172.

[12] Lee, D.X., Xia, W.S., Zhang, J.L. (2008). Enzymatic Preparation of Chitooligosaccharides by Commercial Lipase. Food Chemistry, 111(2): 291-295.
[13] Li, J.F., Wei, F., Dong, X.Y., Guo, L.L., Yuan, G.Y., Huang, F.H., Jiang, M.L., Zhao, Y.D., Li, G.M., Chen, H. (2010). Microwaveassisted Approach for the Rapid Enzymatic Digestion of Rapeseed Meal. Food Science and Biotechnology, 19(2): 463-469.

[14] Warrand, J., Janssen, H.G. (2007). Controlled Production of Oligosaccharides from Amylose by Acid-Hydrolysis under Microwave Treatment: Comparison with Conventional Heating. Carbohydrate Polymers, 69(2): 353-362.

[15] Li, K., Xing, R., Liu, S., Qin, Y., Meng, X., Li, P. (2012). Microwave-assisted Degradation of Chitosan for a Possible Use in Inhibiting Crop Pathogenic Fungi. International Journal of Biological Macromolecules, 51(5): 767773.

[16] Saxena, R.K., Isar, J., Saran, S., Kaushik, R., Davidson, W.S. (2005). Efficient Microwaveassisted Hydrolysis of Triolein and Synthesis of Bioester, Biosurfactant and Glycerides using Aspergillus carneus Lipase. Current Science, 89(6): 1000-1003.

[17] Xia, W., Liu, P., Liu, J. (2008). Advance in Chitosan Hydrolysis by Non-specific Cellulases. Bioresource Technology, 99(15): 67516762 .

[18] Klein, B., Vanier, N.L., Moomand, K., Pinto, V.Z., Colussi, R., da Rosa Zavareze, E., Dias, A.R.G. (2014). Ozone Oxidation of Cassava Starch in Aqueous Solution at Different pH. Food Chemistry, 155:167-173.

[19] Li, J., Du, Y., Liang, H. (2007). Influence of Molecular Parameters on the Degradation of Chitosan by a Commercial Enzyme. Polymer Degradation and Stability, 92(3): 515-524.

[20] Lin, S.B., Lin, Y.C., Chen, H.H. (2009). Low Molecular Weight Chitosan Prepared with the Aid of Cellulase, Lysozyme and Chitinase: Characterisation and Antibacterial Activity. Food Chemistry, 116(1): 47-53.

[21] Mello, P.A., Barin, J.S., Guarnieri, R.A. (2014). Microwave Heating. pp.60-75. In: Microwave-Assisted Sample Preparation for Trace Element Determination. Flores EMM (ed). Elsevier, Amsterdam.

[22] Galema, S.A. (1997). Microwave Chemistry. Chemical Society Reviews, 26(3): 233-238.

[23] Su, P., Wang, S., Shi, Y., Yang, Y. (2013). Application of Cellulase-polyamidoamine Dendrimer-modified Silica for Microwave-assisted Chitosan Enzymolysis. Process Biochemistry, 48(4): 614-619.

[24] Kumar, A.B.V., Varadaraj, M.C., Gowda, L.R., Tharanathan, R.N. (2007). Low Molecular Weight Chitosans-preparation with the Aid of Pronase, Characterization and their 
Bactericidal Activity towards Bacillus Cereus and Escherichia coli. BBA General Subjects, 1770(4): 495-505.

[25] Prasertsung, I., Damrongsakkul, S., Saito, N. (2013). Degradation of B-chitosan by Solution Plasma Process (SPP). Polymer Degradation and Stability, 98(10): 2089-2093.

[26] Li, J., Du, Y., Yang, J., Feng, T., Li, A., Chen, P. (2005). Preparation and Characterisation of Low Molecular Weight Chitosan and ChitoOligomers by a Commercial Enzyme. Polymer Degradation and Stability, 87(3): 441-448.
[27] Singh, J., Dutta, P.K., Dutta, J., Hunt, A.J., Macquarrie, D.J., Clark, J.H. (2009). Preparation and Properties of Highly Soluble Chitosan-L-glutamic Acid Aerogel Derivative. Carbohydrate Polymers, 76(2): 188-195.

[28] Luo, W.B., Han, Z., Zeng, X.A., Yu, S.J., Kennedy, J.F. (2010). Study on the Degradation of Chitosan by Pulsed Electric Fields Treatment. Innovative Food Science and Emerging Technologies. 11(4): 587-591. 